\title{
Optimizing and Improving the Growth Quality of ZnO Nanowire Arrays Guided by Statistical Design of Experiments
}

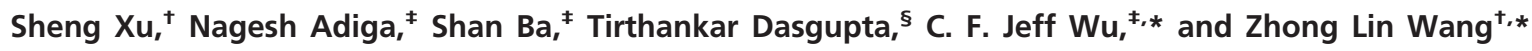 \\ ${ }^{\dagger}$ School of Materials Science and Engineering, Georgia Institute of Technology, 771 Ferst Drive NW, Atlanta, Georgia 30332, ${ }^{\ddagger}$ H. Milton Stewart School of Industrial and

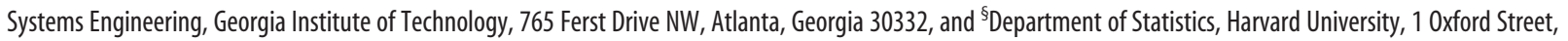 \\ Cambridge, Massachusetts 02138
}

Z nO nanowires (NWs), an outstanding member of the family of onedimensional nanostructures, ${ }^{1-3}$ have found many applications in fabricating electronic, optoelectronic, electrochemical, and electromechanical devices, such as ultraviolet (UV) lasers, ${ }^{4}$ light-emitting diodes, ${ }^{5}$ field emission devices, ${ }^{6,7}$ solar cells, ${ }^{8}$ as well as piezo-nanogenerators. ${ }^{9,10}$ In general, $\mathrm{ZnO}$ NWs are typically manufactured by two methods, namely, physical vapor deposition and hydrothermal decomposition. In contrast to the physical vapor deposition, synthesized via the wet chemical method, the $\mathrm{ZnO}$ NWs are of low aspect ratio typically of 10 to $15,{ }^{11,12}$ which greatly limits their applications in which flexible and high surface to volume ratio $\mathrm{ZnO}$ NWs are needed. There has been a few reports that the aspect ratio of the $\mathrm{ZnO} N W$ s could be increased by adding some external capping agents, such as ethylenediamine $(E n)^{13}$ and polyethylenimine (PEl), ${ }^{8}$ however, at the same time, these capping agents might also introduce some undesirable impurities into the $\mathrm{ZnO}$ NWs, which would greatly undermine their applications for which untainted $\mathrm{ZnO}$ NWs are required.

In most existing literature, synthesis of nanomaterials lacks of theoretical guidance for achieving high quality and reproducible nanomaterials. The practical requirements for nanomaterial synthesis are morphology control, dimensionality control, and orientation control. In this study, as guided by statistical methods, we performed a sequence of experiments toward growing aspect ratio enhanced ZnO NW arrays. Classic statistical analysis did not suit this application due to the uncontrollable variation in the process and complex interactions among various factors. In this work, we use pick-the-winner www.acsnano.org
ABSTRACT Controlling the morphology of the as-synthesized nanostructures is usually challenging, and there lacks of a general theoretical guidance in experimental approach. In this study, a novel way of optimizing the aspect ratio of hydrothermally grown $\mathrm{Zn} 0$ nanowire (NW) arrays is presented by utilizing a systematic statistical design and analysis method. In this work, we use pick-the-winner rule and one-pair-at-a-time main effect analysis to sequentially design the experiments and identify optimal reaction settings. By controlling the hydrothermal reaction parameters (reaction temperature, time, precursor concentration, and capping agent), we improved the aspect ratio of $\mathrm{ZnO} \mathrm{NW}$ s from around 10 to nearly 23. The effect of noise on the experimental results was identified and successfully reduced, and the statistical design and analysis methods were very effective in reducing the number of experiments performed and in identifying the optimal experimental settings. In addition, the antireflection spectrum of the as-synthesized $Z n 0 \mathrm{NWs}$ clearly shows that higher aspect ratio of the $\mathrm{ZnO} \mathrm{NW}$ arrays leads to about $30 \%$ stronger suppression in the UV-vis range emission. This shows great potential applications as antireflective coating layers in photovoltaic devices.

KEYWORDS: ZnO nanowires - aspect ratio - hydrothermal growth - statistical design of experiments (DOE) · pick-the-winner (PW) rule · one-pair-at-a-time main effect analysis

rule $^{14}$ and one-pair-at-a-time main effect analysis ${ }^{15}$ to identify optimal reaction settings. By controlling the hydrothermal reaction parameters, for instance, the precursor concentration, the reaction time, and temperature, the aspect ratio of the $\mathrm{ZnO}$ NWs can be improved to almost twice that of the regular $\mathrm{ZnO}$ NWs. Furthermore, the antireflection properties were also improved dramatically with increased aspect ratio of the ZnO NW arrays, which shows great potential applications as antireflection coating layer for solar cells.

\section{RESULTS AND DISCUSSION}

Reaction Mechanism and Statistical Design of Experiments. The annealing process helped the as-deposited Au layer to form a uniform crystalline thin layer on the surface of the Si substrate, which was critical to the oriented growth of aligned $\mathrm{ZnO} N W s$. The chemistry of the growth has been well-

\section{*Address correspondence to jeffwu@isye.gatech.edu, zlwang@gatech.edu.}

Received for review May 21, 2009 and accepted June 08, 2009.

Published online June 17, 2009. 10.1021/nn900523p CCC: \$40.75

(c) 2009 American Chemical Society 
documented. ${ }^{11,16}$ Zinc nitrate salts provide $\mathrm{Zn}^{2+}$ ions required for building up $\mathrm{ZnO}$ NWs, while water molecules in the solution provide $\mathrm{O}^{2-}$ ions. Even though the exact function of HMTA during the growth is still unclear, it is believed to act as a weak base, which would slowly hydrolyze in the water solution and gradually release $\mathrm{OH}^{-}$. This is critical in the synthesis process because, if the HMTA hydrolyzes too fast and produces a lot of $\mathrm{OH}^{-}$in a short period of time, the $\mathrm{Zn}^{2+}$ ions in solution would precipitate out very quickly due to the high $\mathrm{pH}$ environment, which would have little contribution to the $\mathrm{ZnO}$ NW oriented growth, and eventually results in fast consumption of the nutrient and limits further growth of the $\mathrm{ZnO}$ NWs.

$$
\begin{gathered}
\left(\mathrm{CH}_{2}\right)_{6} \mathrm{~N}_{4}+6 \mathrm{H}_{2} \mathrm{O} \leftrightarrow 4 \mathrm{NH}_{3}+6 \mathrm{HCHO} \\
\mathrm{NH}_{3}+\mathrm{H}_{2} \mathrm{O} \leftrightarrow \mathrm{NH}_{3} \cdot \mathrm{H}_{2} \mathrm{O} \\
\mathrm{NH}_{3} \cdot \mathrm{H}_{2} \mathrm{O} \leftrightarrow \mathrm{NH}_{4}^{+}+\mathrm{OH}^{-} \\
\mathrm{Zn}^{2+}+2 \mathrm{OH}^{-} \leftrightarrow \mathrm{Zn}(\mathrm{OH})_{2} \\
\mathrm{Zn}(\mathrm{OH})_{2} \stackrel{\Delta}{\rightarrow} \mathrm{ZnO}+\mathrm{H}_{2} \mathrm{O}
\end{gathered}
$$

The growth process of the ZnO NWs can be described and controlled through the five chemical reactions listed above. All of the five reactions are actually in equilibrium and can be controlled by adjusting the reaction parameters, such as precursor concentration, growth temperature, and growth time, in order to push the reaction equilibrium forward or backward, which enables us to tune the aspect ratio of the $\mathrm{ZnO}$ nanowires, as shown in Figure 1. In general, precursor concentration determines the NW density and aspect ratios. Growth time and temperature control the $\mathrm{ZnO}$ NW morphology and aspect ratios, as well. In most of the existing literature, there lacks of a systematic study on

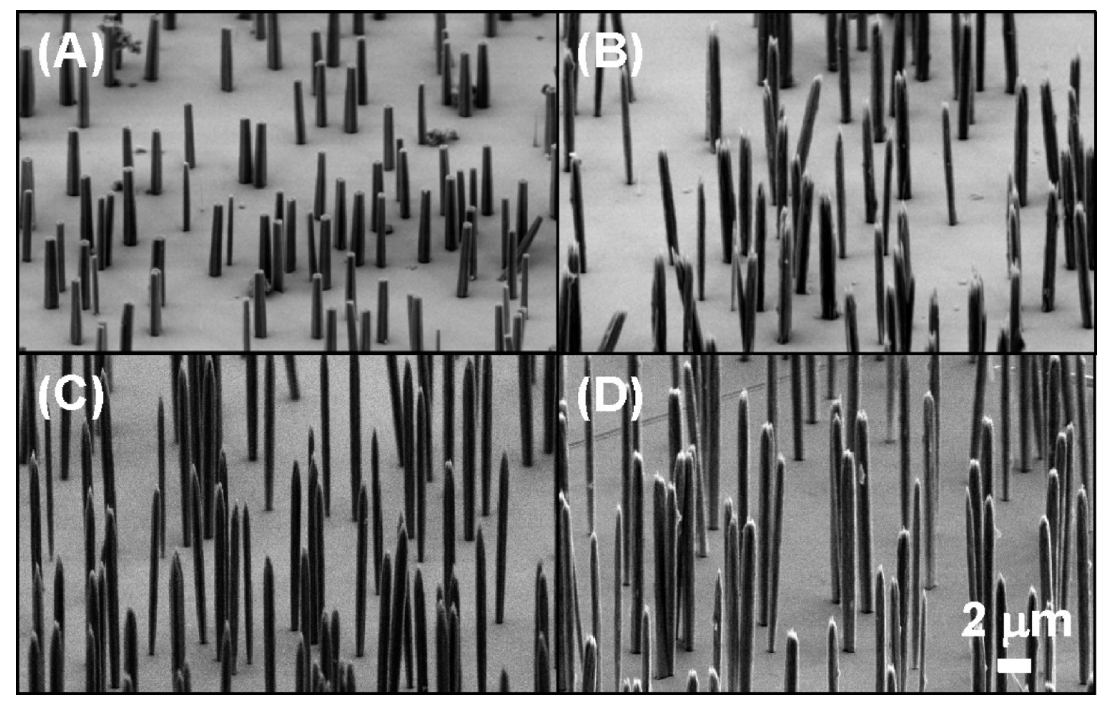

Figure 1. SEM images of $\mathrm{ZnO}$ nanowires with aspect ratio of $(\mathrm{A}) 7.7$, (B) 12.4, (C) 14.5, and (D) 22.3 . the growth conditions and how do they affect the morphologies of the received nanowires. A general approach is "cook and look". Our goal is to use statistical theory to guide our synthesis so that we can systematically achieve high quality, reproducibly desired nanostructures.

The control factors (reaction parameters) that were changed in the course of experiments include the following: (1) Temperature of the furnace (temp), measured in Celsius degrees. (2) Time for which the experimental setup was placed in the furnace (time),

measured in hours. (3) Concentration of the zinc precursor solution (Zn), measured in millimoles per liter. (4) The amount of capping agent that was used in the experiment (capping) was measured in terms of the ratio to the zinc precursor concentration.

Note that the chemistry term "reaction parameter" is often referred to as "experimental factor" in the statistical literature, and in the rest of this paper, these two terms will be used interchangeably. The factors will be varied among different levels. Generally speaking, high temperature and low zinc precursor concentration favor thinner and longer NWs; short reaction time $(<4 \mathrm{~h})$ and long reaction time ( $>48 \mathrm{~h}$ ) decrease the aspect ratio of the $\mathrm{ZnO}$ NWs. Only intermediate reaction time gives a desirable aspect ratio.

The experiments were conducted on a sequential basis. In the first two stages, we tried a wider range of experimental parameters to narrow down the range for good growth of the NWs. Once good yield of NWs was obtained, we concentrated on exploring settings to increase the aspect ratio. When an experiment resulted in NWs with higher aspect ratio, subsequent experiments would be conducted by changing the parameters in the vicinity and toward the optimal setting. If the experiment resulted in NWs with low aspect ratio, the corresponding parameter settings were considered undesirable and the vicinity of these values was avoided in the subsequent experiments. In the end, we also tested the consistency of the results. It is important to get good and reproducible results whenever the experiments are conducted. Hence, in the last stage, we performed the experiments repeatedly to ascertain that the settings chosen were indeed optimal.

\section{Stage I: Analysis from Rudimentary} Experiments. Prior to collaboration with statisticians, a number of preliminary experiments were conducted. SEM images of about 111 samples were available, which are referred to as stage I experiments henceforth. On the basis of these images, we observed good growth in about $60 \%$ of these trials. For many samples, either the NWs grew sparsely or clustered in certain locations. Quite often, 
TABLE 1. Summary of Parameter Ranges of the

Rudimentary Experiments

\begin{tabular}{ll}
\multicolumn{1}{c}{ parameter } & \multicolumn{1}{c}{ settings } \\
\hline temperature of furnace $/{ }^{\circ} \mathrm{C}$ & $65,70,75,80$ \\
growth time $/ \mathrm{h}$ & $3-42$ \\
zinc concentration $/ \mathrm{mM}$ & $0.05-50$ \\
capping agent & additive or no additive \\
\hline
\end{tabular}

they were lying horizontally on the substrate. Among these 111 trials, the highest aspect ratio was found to be around 12. The settings used for these experiments are listed in Table 1.

Here is a summary of how the experimental parameters influenced the growth of NWs:

- Capping agent: Additive acetic ions (Ac) provided higher aspect ratio compared to other additives (sodium dodecyl sulfate (SDS) and ethylenediamine (En)).

-Both the length and diameter of the NWs increased with time.

-Zinc concentration influenced aspect ratio significantly. It was found that higher $\mathrm{Zn}$ values resulted in lower aspect ratio and vice versa.

- Growing NWs for very little time $(3-10 \mathrm{~h})$ resulted in very poor growth and aspect ratio.

- Low temperature $\left(60^{\circ} \mathrm{C}\right)$ did not yield good NWs. Very few experiments were performed at $80^{\circ} \mathrm{C}$.

Stage II: Explore the Experimental Settings. Traditionally, engineers tend to use one-factor-at-a-time approach to plan experiments, wherein settings are changed for only one factor at a time, based on the previous results. This approach is easy to understand but has some drawbacks when compared to the statistical design of experimental methods such as factorial designs.

Full factorial design is a statistical experimental design that consists of all possible combinations of levels for $k$ factors, each taking on two or more levels. ${ }^{15}$ For example, a full factorial design with $k$ factors taking on two levels would comprise all $2^{k}$ combinations (runs). Fractional factorial design is a carefully chosen subset (fraction) of a full factorial design. For example, if we have $k$ factors at two levels each, a $1 / 2^{p}$ fraction of the full factorial design is denoted as $2^{k-p}$ design.

Factorial experiments have some advantages over one-factor-at-time experiments. First of all, they requires less runs for the same precision in effect estimation. Second, dependency of the influence of a factor on the level of another factor can be detected. This is called interaction between the two factors in statistical literature. Third, interpretations and applicability from factorial designs are more general. Last, in case of factorial designs, the chance of missing optimal combination of settings is smaller. Wu and Hamada give the design and analysis strategies of full factorial and fractional factorial designs in detail. ${ }^{15}$

In the previous stage, zinc concentration had shown a clear influence on the aspect ratio of the NWs. This

www.acsnano.org

\begin{tabular}{|c|c|c|c|c|c|}
\hline code & temp & time & $\mathrm{Zn}$ & capping & $\begin{array}{c}\text { growth } \\
\text { (rated on a } 1-5 \text { scale) }\end{array}$ \\
\hline A & 65 & 6 & 2 & $A C$ & poor (1) \\
\hline B & 65 & 12 & 5 & none & $\operatorname{good}(5)$ \\
\hline C & 65 & 48 & 20 & high Ac & poor (1) \\
\hline D & 80 & 6 & 5 & high AC & OK, but not dense (2) \\
\hline$E$ & 80 & 12 & 20 & $A C$ & poor (1) \\
\hline$F$ & 80 & 48 & 2 & none & pyramid/cone shaped (3) \\
\hline G & 94 & 6 & 20 & none & poor (1) \\
\hline H & 94 & 12 & 2 & high Ac & $\operatorname{good}(5)$ \\
\hline | & 94 & 48 & 5 & $A C$ & $\operatorname{good}(5)$ \\
\hline
\end{tabular}

time we performed the experiments with three levels of 2, 5, and $20 \mathrm{mM}$. From the past data, we observed that low temperatures did not provide good NWs. To explore the effect of temperature, we widened the range of the temperature values and chose 65,80 , and $94{ }^{\circ} \mathrm{C}$. Since short growth time resulted in poor growth of NWs, we went with a higher value. The levels chosen for temperature were 6,12 , and $48 \mathrm{~h}$. Note that these values went beyond the range of values used before. It was discovered that the capping agent (additive) Ac provided better NWs (higher aspect ratio) compared to other additives, such as SDS and En. Hence, we continued with the additive Ac for the subsequent experiments. We tested the effect of using capping agent at two levels, in addition to another control level where no capping agent was used. Ac indicates that concentration of the capping agent is the same as the zinc concentration, while high Ac indicates that the concentration of the capping agent is twice the zinc concentration.

Using these four factors at three levels, we designed and conducted the experiments as shown in Table 2, which consisted of nine experiments. This design is referred to as a $3^{4-2}$ fractional factorial design. Here the base 3 refers to the number of levels over which the factors are varied. The exponent 4-2 indicates that four factors are considered, and instead of performing all their $3^{4}$ level combinations, we choose only a $3^{-2}$-th fraction (i.e., 1/9 fraction). The results of these experiments are provided in Table 2, and the corresponding SEM images are shown in the Supporting Information (SI) Figure S1.

From Table 2, we observed that few of the combinations provided good results. For some of the settings, growth of NWs was very poor, hence measuring the aspect ratio was meaningless in these cases. Instead, we rated them according to the level of growth of the NWs in the sample. "Poor" refers to the sample where NWs were either absent or clustered at a few places. "Good" refers to samples where the NWs were upright and dense. Some of the aspects which were considered to reduce the effectiveness of the NWs were slantness of the NWs, pyramid/conical shape of the NWs, and very 


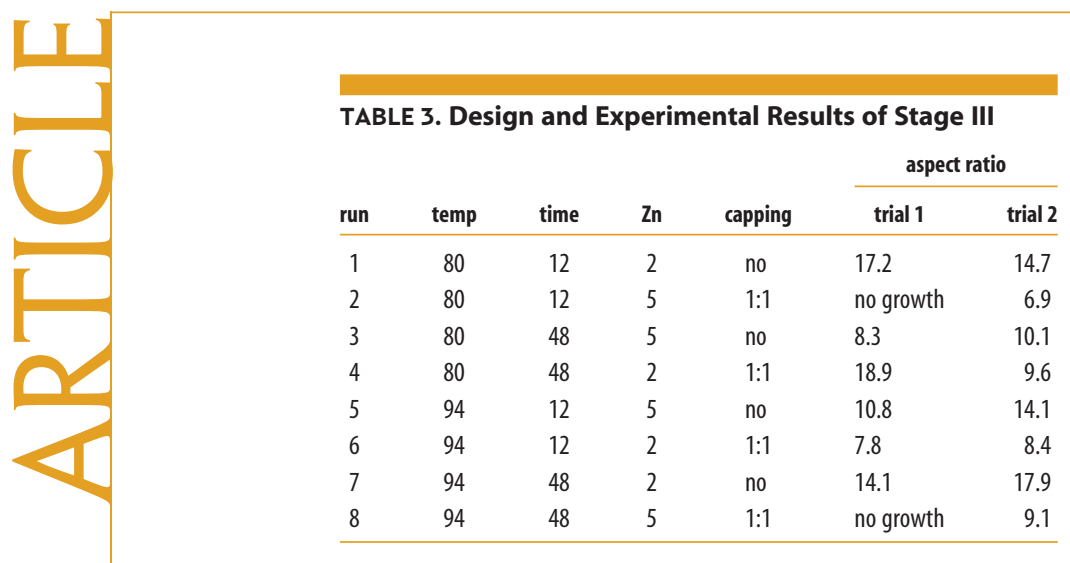

low density of the NWs (i.e., smaller number of NWs per unit surface area).

From Table 2, we observed that high zinc concentration $(20 \mathrm{mM})$ resulted in poor growth, irrespective to the levels of other factors. Chances of good growth were higher when the NWs were grown for 12 or $48 \mathrm{~h}$. Similarly, when temperature was set at 80 or $94^{\circ} \mathrm{C}$, the chance of good growth was also higher. On the basis of these observations, we ignored the level of temperature at $65^{\circ} \mathrm{C}$, time at $6 \mathrm{~h}$, and zinc concentration at 20 $\mathrm{mM}$, which led to poor growth. Our focus was narrowed down to two other good settings. The influence of the capping agent was not clear from the above experiments, possibly because the changes in other factor settings may have overshadowed the effect of the capping agent. Therefore, in the next stage, we dropped the "high Ac" level for capping to minimize the side effects it may cause, such as change of ionic strength and $\mathrm{pH}$ value of solution.

Stage III Experiments: Narrow down the Focus. During this stage, we performed the experiments as shown in Table 3. This design is referred to as a $2^{4-1}$ fractional factorial design since only half (i.e., $2^{-1}$ ) of the $2^{4}$ runs for four factors at two levels were conducted. We replicated these experiments twice (trials 1 and 2 in Table 3) to improve accuracy. The results of the experiment are provided in Table 3, and the corresponding SEM images are shown in SI Figures S2 and S3. For each sample, we measured the lengths and widths of around 20 NWs by Photoshop and then calculated their aspect ratios average.

It was evident that most of the experiments resulted in good growth of NWs. Moreover, the average aspect ratio was also improved when compared to the previous stages. However, the experimental results over the two trials were not always consistent. Some runs were consistent (such as runs 1 and 7, which resulted in high aspect ratio both times, and runs 3 and 6 , which yielded low aspect ratio both times), but many other runs were not. For instance, run 4 yielded the highest aspect ratio in the first trial but not so well in the second trial. For runs 2 and 8, trial 1 resulted in very poor or no growth, while trial 2 resulted in good growth (al-

TABLE 4. Experimental Results for the Three Trials of Stage III

\begin{tabular}{clcl} 
& \multicolumn{3}{c}{ aspect ratio } \\
\cline { 2 - 4 } run & trial 1 & trial 2 & trial 3 \\
\hline 1 & 17.2 & 14.7 & 13.6 \\
2 & no growth & 6.9 & 9.9 \\
3 & 8.3 & 10.1 & 17.7 \\
4 & 18.9 & 9.6 & 28.4 \\
5 & 10.8 & 14.1 & 15.0 \\
6 & 7.8 & 8.4 & 11.2 \\
7 & 14.1 & 17.9 & 18.8 \\
8 & no growth & 9.1 & no growth
\end{tabular}

though low aspect ratio). Possible reasons for these inconsistencies will be discussed later.

Due to these inconsistencies, we continued to perform a third trial for this stage. Results for all three trials are shown in Table 4. Comparing results for each run from the three trials, we can see that, in general (except for the first and eighth run), the aspect ratio for the third trial was higher than the first and second trials. Furthermore, the order of runs based on the aspect ratio of the NWs remained almost the same for all three trials.

In this stage, we were able to obtain good growth with reasonable high aspect ratio. Next we move toward obtaining consistently higher aspect ratio. However, there were some challenges. As discussed before, some experiments were not quite reproducible. Their results varied a lot when performed at different times, as shown in Table 4. The inconsistency was due to the presence of some uncontrollable or unknown noise factors during the growth process. Because of this, traditional statistical modeling may not be applicable since the noise variation can sometimes be so large as to overshadow the effect of the controlled parameter settings. Hence, obtaining a prediction model that can predict the result under given parameter settings was difficult. In view of this, the huge variation among the results in stage III led to another motivation of our study: to reduce the effect of noise factors on the output. In other words, we needed to find parameter settings that not only produce NWs with higher aspect ratio but also give consistently good results.

Sources of Noise during the Growth of NWs, Control Strategies, and Data Analysis Approaches. In order to reduce the noise, we performed a cause and effect analysis. By analyzing the effects at each step, we try to enumerate the causes for uncontrolled variation and list the possible causes of noise.

The major causes of the uncontrolled noise appeared to originate from the deposition of $\mathrm{Ti}$ and $\mathrm{Au}$ over the substrate by plasma sputtering. During this complex process, we had control only over the power of the plasma sputtering, which was usually fixed by experience. However, due to technical difficulties, the variation of working temperature inside the chamber 
was uncontrollable (and cannot be measured). In statistical literature, this type of uncertainty is referred to as internal noise. ${ }^{15}$ Although the change of working power of the plasma sputtering may have an effect on the temperature, we do not know their relationship clearly. Hence, it is not possible to indirectly control the temperature via the change of working power.

The second source of the noise was observed to be due to human interference, referred to as human disturbance factors. Typical examples included disturbance to the furnace by some other users during the growth of NWs and unexpected accidents while handling the samples, etc. Although this kind of accident was unusual, it could account for a few very inconsistent results. A typical example was run 4 in Table 4 . This run yielded the highest aspect ratio in the first and third trials but gave a very poor result in the second trial. Here we can reasonably argue that its growth in the second trial was affected by some unexpected disturbances or accidents, and therefore, we still regarded the corresponding experimental setting as the optimal one in stage III, based on its optimal results in the first and third trials. Note that the "human noise" of this kind could only damage the growth of NWs.

The third source contains all the other types of noise, known or unknown, including the environmental factors like humidity, surface condition of the substrate, etc. While this type of noise was not fully under our control, we can sometimes partly control them or nullify their effect.

To reduce these three types of noise, some additional steps were taken in the subsequent experiments. First, a large amount of substrates were prepared at one time for the future experiments. In this way, the effect of the noise during the deposition of metals was eliminated. Second, subsequent experiments were scheduled during periods such as weekends or holidays, when there was much less disturbance to the furnace or other equipment. By taking these steps, we tried to minimize the effect of noise, if not completely removing it.

Also note that, within each stage (or each trial), all eight or nine experiments were grouped and performed together to form a block. In the design of experiment, a group of homogeneous units is referred to as a block, such that the within-block variation is much smaller than the between-block variation. ${ }^{15}$ On the basis of the principle of blocking, the comparison of responses in the same block was reasonable because the effect of noise on them was more or less similar and got canceled out. However, since the noise factors usually changed over time, the comparison among results from different blocks was meaningless. For example, within each of the eight experiments in trial 1 or 3 of stage III, we found the factor settings in run 4 were optimal. However, comparison between results in these two trials would not give any useful information, except that
TABLE 5. Design and Experimental Results of Stage IV

\begin{tabular}{cccccc} 
run & temp & time & Zn & capping & aspect ratio \\
\hline 1 & 80 & 24 & 1 & no & 23.4 \\
2 & 80 & 24 & 3 & $1: 1$ & 23.0 \\
3 & 80 & 48 & 3 & no & 10.6 \\
4 & 80 & 48 & 1 & $1: 1$ & 19.7 \\
5 & 94 & 24 & 3 & no & 17.0 \\
6 & 94 & 24 & 1 & $1: 1$ & 16.5 \\
7 & 94 & 48 & 1 & no & 14.3 \\
8 & 94 & 48 & 3 & $1: 1$ & 10.1
\end{tabular}

the noise has significantly changed in these two blocks of experiments.

Due to the presence of large noise and inconsistent results of the three trials, classic statistical approaches like the main effect analysis ${ }^{15}$ would not be suitable here. In this stage, we adopted a nontraditional strategy called pick-the-winner ${ }^{14}$ and focused on picking new settings at or around the optimal settings previously observed. The traditional main effect analysis, which is based on the average of the response data, could be misleading when data have large noise or when complex interactions exist among factors while the paucity of data does not allow the interactions to be fully analyzed. However, under these circumstances, the pick-the-winner strategy could still identify optimal settings effectively and efficiently, as will be seen later. Another justification for using pick-the-winner is that good growth can only result from "good settings". Random disturbance may result in poor growth for the corresponding experiment but not optimal growth of NWs, based on which pick-the-winner rule works.

In stage III, zinc concentration was found to be the major factor affecting the aspect ratio. Since zinc concentration at $2 \mathrm{mM}$ outperformed that at $5 \mathrm{mM}$, we tried additional settings around it: 1 and $3 \mathrm{mM}$ for the next stage. Since $48 \mathrm{~h}$ was better than $12 \mathrm{~h}$, for the next stage, we set time at 24 and $48 \mathrm{~h}$ to check if there will be any improvement at $24 \mathrm{~h}$. Since we did not see clear advantage of one temperature setting over another, we would retain the two levels of the temperature as before, 80 and $94^{\circ} \mathrm{C}$. Similarly, since we did not observe much effect of the capping agent, we retained the two settings as before.

Stage IV Experiments and Results. A $2^{4-1}$ design was conducted for the selected levels of the four factors as discussed before. The results of these experiments are shown in Table 5, and the corresponding SEM images are shown in SI Figure S4.

It was observed that the aspect ratios in this stage have become uniformly high. They have been much more consistent and greatly improved than those in previous stages. This improvement may be due to reduced uncontrolled noise for this stage and choice of good settings. However, we would not compare the results with previous stages directly due to the noise. The improvements in aspect ratio were large enough 
A

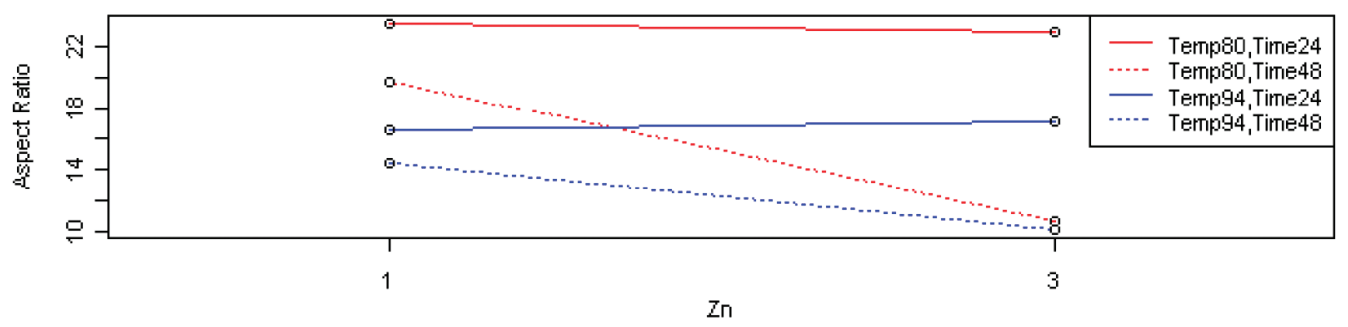

B



c

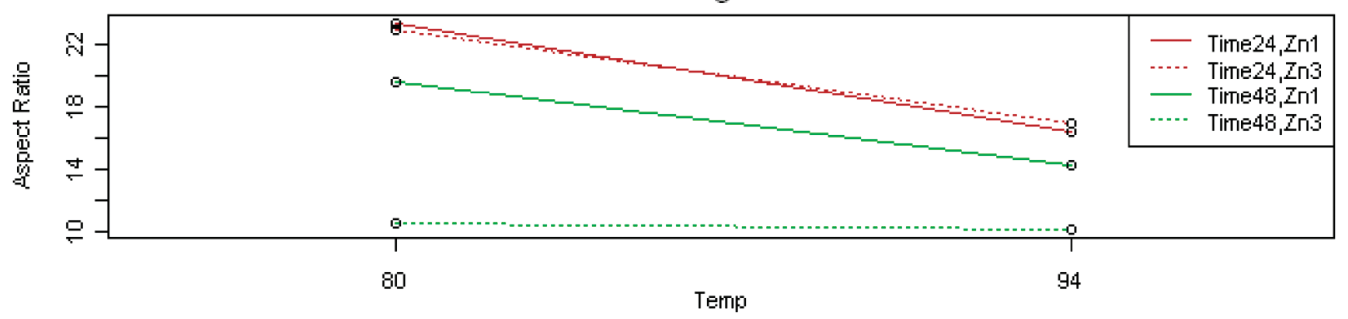

Figure 2. One-pair-at-a-time main effect analysis for each pair of factors in stage IV.

to give us confidence that we were on the right track. We can still make comparison between different setting combinations within this stage.

It could be seen that the effect of capping agent was not significant in this stage, so it was omitted in the following analysis. To identify good factor settings, traditional main effect analysis is not suitable due to possible noise and complex interactions among factors. Here we introduced a slight variation, called onepair-at-a-time main effect analysis. In this method, the responses are grouped into pairs, wherein level of only one factor is changed. This could facilitate our analysis of the main effect in the presence of complex interactions and possible noise. We use Figure 2 to illustrate this method. Plots A, B, and C in Figure 2 were used to compare the two chosen levels for zinc concentration, time, and temperature separately. In each plot, there are eight points corresponding to the eight runs in Table 5, and the vertical axis shows the aspect ratio of the corresponding experiment. In each plot, the four lines represent that the points connected by them are having the same settings for the other two experimental factors. In other words, the settings for the points on the same line are different only in the level of factor being compared in the plot. For example, in plot A of Figure 2 , the two points connected by the red line have the same settings of temperature $\left(80^{\circ} \mathrm{C}\right)$ and time $(24 \mathrm{~h})$ but differ in zinc concentration at 1 and $3 \mathrm{mM}$. Note that this main effect analysis method can be easily modified to be one-group-at-a-time when the factor being analyzed has more than two levels. We will encounter these situations in later stages.

From the three plots in Figure 2, we can easily interpret all the main effects for the three factors ( $Z n$, time, temp) and their possible interactions. Here, for zinc concentration, plot A clearly shows that $1 \mathrm{mM}$ was better than $3 \mathrm{mM}$. Similarly, plot B identifies that $24 \mathrm{~h}$ yielded higher aspect ratio than $48 \mathrm{~h}$; and from plot $\mathrm{C}, 80^{\circ} \mathrm{C}$ was slightly better than $94^{\circ} \mathrm{C}$.

Stage V Experiments and Results. On the basis of the observations from the previous sets of experiments, we moved on to design a new set of experiments for stage V. Recall that in stage III $2 \mathrm{mM}$ for zinc concentration was better than $5 \mathrm{mM}$, and in stage IV, the zinc concentration of $1 \mathrm{mM}$ yielded higher aspect ratio than did 3 $\mathrm{mM}$. However, due to the systematic change of noise among different stages, we cannot compare the levels of 1 and $2 \mathrm{mM}$ directly. Therefore, for stage $V$, it was a good idea to compare these two levels directly. Also, we decided to choose one additional level for zinc concentration at $0.5 \mathrm{mM}$ to explore the promising left side of $1 \mathrm{mM}$. Thus, the three levels we choose for zinc concentration in stage $V$ were $0.5,1$, and $2 \mathrm{mM}$. As for time, in stage IV, NWs grown for $24 \mathrm{~h}$ had higher aspect ratio than those grown for $48 \mathrm{~h}$. Also, in stage III, $48 \mathrm{~h}$ yielded higher aspect ratio than $12 \mathrm{~h}$. Hence, in stage $\mathrm{V}$, we 


\begin{tabular}{|c|c|c|c|c|c|}
\hline run & temp & time & $\mathrm{Zn}$ & capping & aspect ratio \\
\hline 1 & 80 & 24 & 0.5 & no & 14.5 \\
\hline 2 & 80 & 24 & 1 & no & 17.0 \\
\hline 3 & 80 & 24 & 2 & no & 9.3 \\
\hline 4 & 80 & 36 & 0.5 & $1: 1$ & 20.2 \\
\hline 5 & 80 & 36 & 1 & $1: 1$ & 18.9 \\
\hline 6 & 80 & 36 & 2 & $1: 1$ & 20.0 \\
\hline 7 & 94 & 24 & 0.5 & $1: 1$ & 7.0 \\
\hline 8 & 94 & 24 & 1 & $1: 1$ & 13.7 \\
\hline 9 & 94 & 24 & 2 & $1: 1$ & 10.5 \\
\hline 10 & 94 & 36 & 0.5 & no & 11.4 \\
\hline 11 & 94 & 36 & 1 & no & 15.0 \\
\hline 12 & 94 & 36 & 2 & no & 13.9 \\
\hline
\end{tabular}

chose 24 and $36 \mathrm{~h}$ as the two levels for growing time. In stage IV, lower temperature $\left(80^{\circ} \mathrm{C}\right)$ provided higher aspect ratio than higher temperature $\left(94^{\circ} \mathrm{C}\right)$. However, in stage III, the same settings were not equally good, and the advantage of 80 over $94^{\circ} \mathrm{C}$ was not evident. Therefore, in stage $\mathrm{V}$, we would retain the same settings for further comparison. Since the effect of the capping agent was not detected in the previous stages, we can reasonably assume that the effect of capping agent on the aspect ratio is negligible and can be dropped from the study. However, in stage $V$, we would give another try to vary the capping agent as before.

The new set of experiments for stage $V$ contained 12 runs, and was a $2^{2} 3^{1}$ full factorial design including all possible combinations of the levels for time, temperature, and zinc concentration. Note that the column for capping is not a part of the $2^{2} 3^{1}$ design. The settings of capping are chosen such that all possible level combinations of capping temperature and capping time appear equally often. This particular property of the design is referred to as orthogonality. ${ }^{15}$ This set of experiments and the corresponding results are shown in Table 6. The SEM images are in SI Figure S5. Note that, in this stage, the levels for temperature and capping agent have not been changed, and we have also retained one level for zinc concentration (1 $\mathrm{mM}$ ) and one level for time ( $24 \mathrm{~h}$ ) as in stage IV. As a result, we have two common runs between stages IV and $V$, which are Zn, time, temp, capping $=1,24,80$, no and 1, 24, 94, 1:1, respectively. With these two common runs, we would be able to get an idea of the systematic change between these two successive stages.

From the above results, we first compared the two common runs (runs 2 and 8 in Table 6) with runs 1 and 6 in stage IV. The aspect ratios for these two runs were 23.4 and 16.5 in stage IV, and 17.0 and 13.7 for stage $V$. It can be observed that, from stage IV to stage $\mathrm{V}$, the aspect ratio is reduced by around $20-30 \%$. This strengthened our belief that the noise changed systematically over stages (i.e., over time), and we should focus on comparing the parameter settings within each stage.
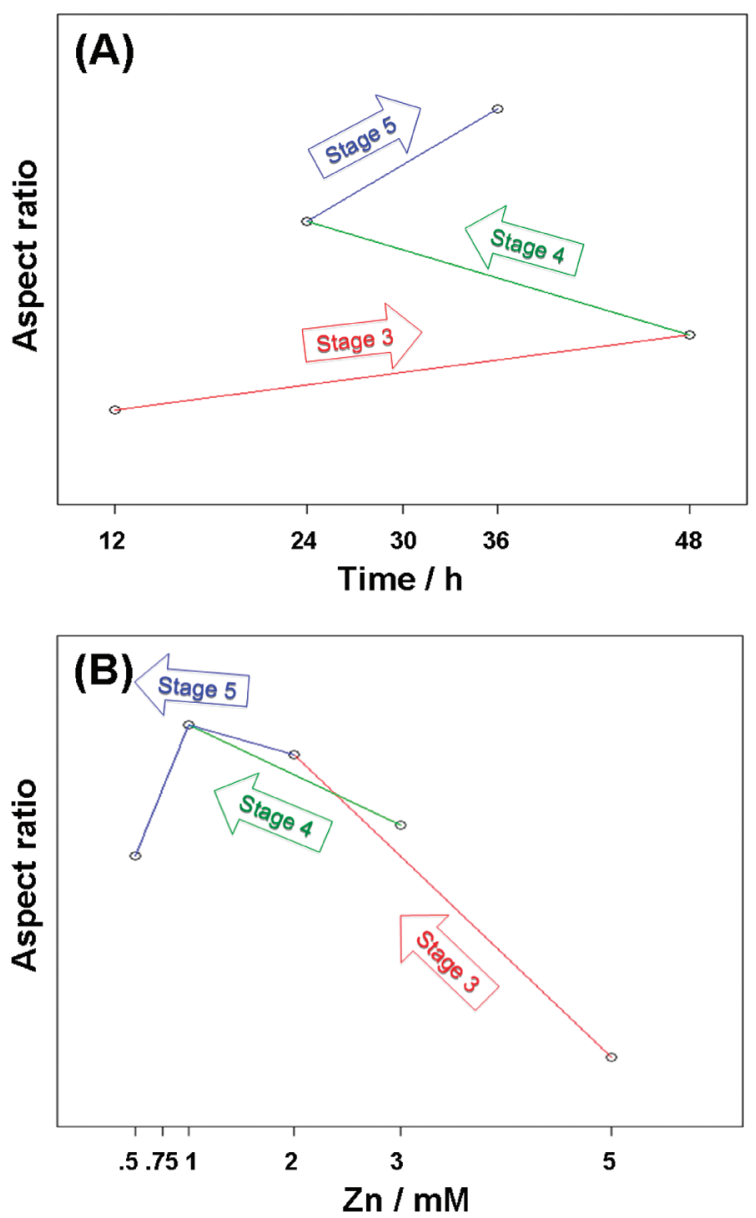

Figure 3. (A) Chang of levels for time in stages III, IV, and V. (B) Change of levels for zinc concentration in stages III, IV, and $\mathrm{V}$.

As in the previous stages, capping agent did not show any effect on the aspect ratio. Hence, this factor was dropped in the subsequent stages of experiments. For the other factors in this stage, we use one-pair-at-atime main effect analysis, or one-group-at-a-time, when the factor being analyzed has multiple levels. The corresponding main effect analyses Figures $\mathrm{S} 6-\mathrm{S} 8$ are provided in SI. It can be seen that the best level of zinc concentration at this stage was $1 \mathrm{mM}$ since, under the same levels for the other factors, the zinc concentration at $1 \mathrm{mM}$ resulted in much higher aspect ratio than the other two levels $(0.5$ and $2 \mathrm{mM})$ in three out of four cases and still did not lose much in its only inferior case. For the time factor, aspect ratio was higher when the NWs were grown for $36 \mathrm{~h}$ than for $24 \mathrm{~h}$. For the temperature, we clearly observed that $80^{\circ} \mathrm{C}$ was better than $94^{\circ} \mathrm{C}$. This was consistent with what we observed in stage IV, and by now, the effect of temperature can be ascertained.

Stage VI Experiments and Results. Before choosing new levels for stage VI experiments, let us first summarize the factor levels we have compared in the previous stages. These comparisons for factors of zinc concentration and time are illustrated in Figure 3. 


\begin{tabular}{ccccc}
\multicolumn{5}{r}{ TABLE 7. Design and Experimental Results of Stage VI } \\
run & temp & time & Zn & aspect ratio \\
\hline 1 & 80 & 30 & 0.75 & 8.3 \\
2 & 80 & 30 & 1 & 19.9 \\
3 & 80 & 30 & 1.5 & 19.6 \\
4 & 80 & 36 & 0.75 & 15.8 \\
5 & 80 & 36 & 1 & 15.1 \\
6 & 80 & 36 & 1.5 & 18.9 \\
7 & 85 & 30 & 0.75 & 14.8 \\
8 & 85 & 30 & 1 & 7.7 \\
9 & 85 & 30 & 1.5 & 16.9 \\
10 & 85 & 36 & 0.75 & 12.4 \\
11 & 85 & 36 & 1 & 17.3 \\
12 & 85 & 36 & 1.5 & 14.3 \\
13 & 90 & 30 & 0.75 & 14.1 \\
14 & 90 & 30 & 1 & 19.6 \\
15 & 90 & 30 & 1.5 & 17.0 \\
16 & 90 & 36 & 0.75 & 16.4 \\
17 & 90 & 36 & 1 & 17.3 \\
18 & 90 & 36 & 1.5 & 18.7 \\
19 & 80 & 36 & 2 & 15.9 \\
\hline
\end{tabular}

On the basis of the discussions and comparisons made above, we chose the zinc concentration at three levels $(0.75,1,1.5 \mathrm{mM})$ to further explore the vicinity of the optimal setting of $1 \mathrm{mM}$. We retained the setting of $1 \mathrm{mM}$ common with the previous stage to assist in comparison. We also tried out the new growth time of $30 \mathrm{~h}$ to compare with the previous optimal $36 \mathrm{~h}$, in an attempt to find out if there is a peak between 24 and $36 \mathrm{~h}$. In the previous stages, it was clear that $80^{\circ} \mathrm{C}$ yielded higher aspect ratio than did $94^{\circ} \mathrm{C}$. Our previous experience also showed that low temperature does not favor good growth. So this time we varied and compared temperature at three levels in the right neighborhood of this optimal setting $\left(80,85\right.$, and $\left.90^{\circ} \mathrm{C}\right)$, with 80 ${ }^{\circ} \mathrm{C}$ being common with the previous stages. The capping agent factor was ignored for this stage and would not be used for the experiments.

Thus, for stage $\mathrm{VI}$, we would use a $2^{1} 3^{2}$ full factorial design with a total of 18 runs. Note that this time we retained one common run $\left(80^{\circ} \mathrm{C}, 36 \mathrm{~h}, 1 \mathrm{mM}\right)$ with stage $\mathrm{V}$. To gain a better understanding of the systematic change over stages $\mathrm{V}$ and $\mathrm{VI}$, we added another common run $\left(80^{\circ} \mathrm{C}, 36 \mathrm{~h}, 2 \mathrm{mM}\right)$ in this stage. This particular setting was chosen because, in previous stage, we had observed that it provided very good results, though not all the chosen levels were optimal. The final set of experiments and results for stage $\mathrm{VI}$ are shown in Table 7. The corresponding SEM images are in SI Figure S9.

Once again, there was a systematic change in the aspect ratio between stage $\mathrm{V}$ and stage VI. The aspect ratio had dropped by $20 \%$, as seen from the two common runs. Also, we noted that run 1 and run 8 yielded unusually low aspect ratios. We considered these two individual runs to have been affected by some unexpected strong disturbance or noise during the grow-
TABLE 8. Reproducibility of the Good Results in Stage VII

\begin{tabular}{ccclccc} 
run & temp & time & Zn & trial 1 & trial 2 & trial 3 \\
\hline 1 & 80 & 24 & 1 & 7.7 & 17.5 & 13.1 \\
2 & 80 & 36 & 2 & 6.2 & 20.5 & 17.9 \\
3 & 80 & 36 & 0.5 & 12.8 & 19.1 & 12.6 \\
4 & 80 & 36 & 1 & 11.4 & 18.3 & 20.1 \\
5 & 80 & 36 & 1.5 & 8.1 & 17.8 & 12.9 \\
6 & 90 & 36 & 1.5 & 6.8 & 18.3 & 17.1 \\
7 & 90 & 30 & 1 & 8.7 & 16.3 & 14.5 \\
8 & 80 & 30 & 1.5 & 7.8 & 18.4 & 20.9 \\
9 & 80 & 30 & 1 & 9.4 & 19.3 & 22.3
\end{tabular}

ing process. Therefore, we treated them as outliers and omitted them in the analysis.

The one-pair(group)-at-a-time main effect analyses Figures $\mathrm{S} 10-\mathrm{S} 12$ for this stage are provided in SI. After we dropped these two points, we observed that this time the aspect ratio corresponding to the three different levels of zinc concentration was close, indicating that we had narrowed down our level range for zinc concentration near the peak since these three levels had already been very close to each other. Also, for the time factor, after the drop of two runs, both 30 and $36 \mathrm{~h}$ seemed to be equally good, with $30 \mathrm{~h}$ only slightly better on average. In the same way, the temperatures of 80 and $90^{\circ} \mathrm{C}$ were equally good, and slightly better than $85^{\circ} \mathrm{C}$. By now, we have closed in the optimal region. After the drop of two outlier values, the resulting aspect ratios were close. In addition, for each factor, there was no obvious pattern that indicates any level had clear advantage over the others. Therefore, for the next stage, we focus on the reproducibility of good results.

Stage VII: Reproducibility Test. In previous stages, we found optimal settings for the factors. In this stage, we focused on ascertaining that these good results are reproducible. On the basis of previous results, we chose nine "winner" settings that had yielded high aspects ratios. Then we performed the whole group of nine experiments at three different times (trials 1, 2, and 3 in Table 8 ) in order to test their reproducibility. The nine chosen settings and the results of three trials are shown in Table 8. The corresponding SEM images are in SI Figures S13-S15.

From Table 8, the second and third trials yielded consistently high aspect ratios. However, results for the first trial were about half of these values. Note that all the experiments for each trial were grouped as a block and conducted in the same time period, while these three trials were conducted at three different times. These results confirmed the systematic change of noise effect over time. The consistently high aspect ratio in trials 2 and 3 showed that our chosen settings were indeed optimal and reproducible.

Applications as Antireflection Coating Layer. Antireflection coating layers play a significant role in enhancing the efficiency of photovoltaic devices by increasing the 
light coupled into the active region of the devices. ${ }^{17-20}$ The intricate distribution of the $\mathrm{ZnO} N W s$ and their textured surface could trap photons, leading to a broadband suppression in reflection. Lee et al. reported that highly textured $\mathrm{ZnO}$ nanorod arrays (NRAs) synthesized via low temperature solution growth played a significant role in the performance of photovoltaic devices as an antireflective coating. ${ }^{20}$ By changing the growth conditions, they modified the shape of the $\mathrm{ZnO}$ nanorod tips, leading to continuously varied refractive index profiles in a single layer. Accordingly, we expect that, as we increase the aspect ratio of the $\mathrm{ZnO} N W$ s combined with the nanoscale surface roughness of the NW arrays, there would be better chances for the incident photons interacting with the NWs' surfaces and therefore the absorption cross section would be considerably larger. Thus, there would be more photons coupled into the ZnO NW and eventually increase the efficiency of the photovoltaic devices.

We measured the antireflection spectra of different aspect ratios of the $\mathrm{ZnO} N W$ arrays at an incident angle of $60^{\circ}$. As illustrated in Figure 4, curves A, B, C, and D correspond to ZnO NWs of aspect ratio of 15.1, 20.1, 20.9, and 22.3, respectively. These four NW arrays have approximately the same NW density, as shown in their SEM images. Obviously, we can see that the ZnO NW arrays with an aspect ratio of 22.3 have an almost $30 \%$ stronger capability of suppressing the reflection than those with an aspect ratio of 15.1 in both the ultraviolet and visible range. That is simply because long NWs would have a much higher chance of interacting and coupling the light photons with the NWs' surface than the short ones. The little bumps at around $900 \mathrm{~nm}$ on the curve come from the detector change during the date collection of the equipment.

\section{CONCLUSION}

With the guidance of a systematic statistical design and analysis, we optimized the aspect ratio of ZnO NWs by controlling the hydrothermal reaction parameters (reaction temperature, time, precursor concentration, and capping agent). The effect of noise on the experimental results was identified and successfully reduced, and the statistical design and analysis methods were very effective in reducing the number of experiments performed and in identifying the optimal experimental settings. We found that, with
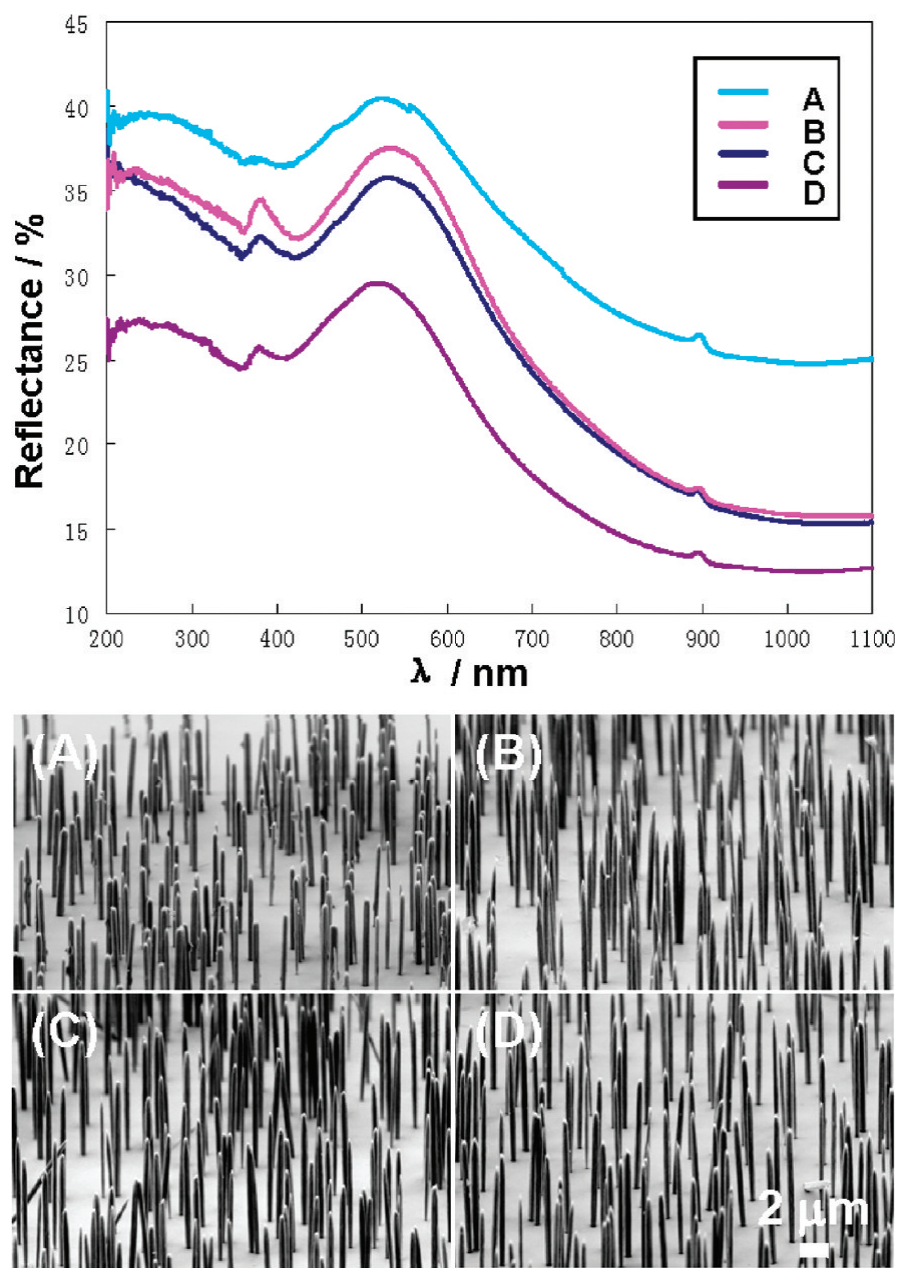

Figure 4. Reflectance spectra of the ZnO NW arrays of different aspect ratio. Curves A, B, C, and D correspond to aspect ratios of 15.1, 20.1, 20.9, and 22.3, respectively, and their representative SEM images.

the precursor concentration in the vicinity of $1 \mathrm{mmol} / \mathrm{L}$, at a reaction temperature of around $80^{\circ} \mathrm{C}$, for about $30 \mathrm{~h}$, the aspect ratio of the $\mathrm{ZnO}$ NW arrays has the highest value of nearly 23 . Those aspect ratio enhanced ZnO NW arrays have great potential applications as antireflective coating layers in photovoltaic devices. The antireflection spectrum of the as-synthesized $\mathrm{ZnO}$ NWs clearly shows that higher aspect ratio of the $\mathrm{ZnO}$ NW arrays leads to stronger suppression in the UV-vis range. This gives a concrete and convenient way to increase the efficiency of photovoltaic devices, such as solar cells.

\section{METHODS}

The NWs were grown on (100) oriented Si wafers. The Si substrate was cleaned by a standard cleaning process. First, the wafer was ultrasonicated consecutively in acetone, ethanol, IPA (isopropyl alcohol), and deionized water each for $10 \mathrm{~min}$, blown dry by nitrogen, and then baked on a hot plate at $200^{\circ} \mathrm{C}$ for 5 min to get rid of any adsorbed moisture, which would otherwise weaken the adhesion of the $\mathrm{Ti}$ and Au layers to the Si substrate. Then, a $50 \mathrm{~nm}$ thick layer of Au was deposited on top of the Si wafer by direct current magnetron plasma sputtering, which is expected to act as an intermediate layer to buffer the lattice mismatch between the $\mathrm{Au}(111)$ surface and the $\mathrm{ZnO}(0001)$ surface to assist the growth. Between the Si wafer and the Au layer, 20 $\mathrm{nm}$ of Ti was deposited as an adhesion layer to increase the adhesion between the $\mathrm{Si}(100)$ surface covered with native oxide and Au film. Then the substrate was annealed at $300{ }^{\circ} \mathrm{C}$ for $1 \mathrm{~h}$ to increase the crystallinity of the Au thin film. The reaction nutrient solution was composed of a 1:1 ratio of zinc nitrate hexahy- 
drate and hexamethylenetetramine (HMTA). Both chemicals were reagent grade from Fluka. The precursor concentration was adjusted from $\sim 1$ to $\sim 100 \mathrm{mM}$ to study its effect on the aspect ratio of the $\mathrm{ZnO}$ NWs. Different levels of both reaction temperature and time were also systematically studied. During the growth, the substrate was put face down at the top of the nutrient solution surface and was able to float at the top of the solution surface by virtue of surface tension of the nutrient solution. ${ }^{16}$ Growth of ZnO NWs was conducted in a mechanical convection oven (Yamato DKN400). The products were examined at $5 \mathrm{kV}$ using a LEO 1550 scanning electron microscope (SEM).

Acknowledgment. Research was supported by NSF (DMS 0706436, CMMI 0403671), DARPA (Army/AMCOM/REDSTONE AR, W31P4Q-08-1-0009), and BES DOE (DE-FG02-07ER46394).

Supporting Information Available: Representative SEM images of the samples in stages from II to VII experiments. One-pair (group)-at-a-time main effect analysis figures for zinc concentration, time, and temperature in stages $\mathrm{V}$ and $\mathrm{VI}$. This material is available free of charge via the Internet at http://pubs.acs.org.

\section{REFERENCES AND NOTES}

1. Wang, Z. L. ZnO Nanowire and Nanobelt Platform for Nanotechnology. Mater. Sci.Eng. Rep. 2009, 64, 33-71.

2. Wang, Z. L. Theme Issue: Inorganic Nanotubes and Nanowires. J. Mater. Chem. 2009, 19, 826-827.

3. Wang, Z. L. Oxide Nanobelts and Nanowires: Growth, Properties and Applications. J. Nanosci. Nanotechnol. 2008, $8,27-55$.

4. Huang, M. H.; Mao, S.; Feick, H.; Yan, H. Q.; Wu, Y. Y.; Kind, H.; Weber, E.; Russo, R.; Yang, P. D. Room-Temperature Ultraviolet Nanowire Nanolasers. Science 2001, 292, 1897 1899.

5. Sun, X. W.; Huang, J. Z.; Wang, J. X.; Xu, Z. A ZnO Nanorod Inorganic/Organic Heterostructure Light-Emitting Diode Emitting at $342 \mathrm{~nm}$. Nano Lett. 2008, 8, 1219-1223.

6. Bai, X. D.; Wang, E. G.; Gao, P. X.; Wang, Z. L. Measuring the Work Function at a Nanobelt Tip and at a Nanoparticle Surface. Nano Lett. 2003, 3, 1147-1150.

7. Wang, X. D.; Zhou, J.; Lao, C. S.; Song, J. H.; Xu, N. S.; Wang, Z. L. In Situ Field Emission of Density-Controlled ZnO Nanowire Arrays. Adv. Mater. 2007, 19, 1627-1631.

8. Law, M.; Greene, L. E.; Johnson, J. C.; Saykally, R.; Yang, P. D. Nanowire Dye-Sensitized Solar Cells. Nat. Mater. 2005, 4, 455-459.

9. Wang, X.; Song, J.; Liu, J.; Wang, Z. L. Direct-Current Nanogenerator Driven by Ultrasonic Waves. Science 2007, 316, 102-105.

10. Wang, Z. L.; Song, J. H. Piezoelectric Nanogenerators Based on Zinc Oxide Nanowire Arrays. Science 2006, 312, 242-246.

11. Vayssieres, L. Growth of Arrayed Nanorods and Nanowires of ZnO from Aqueous Solutions. Adv. Mater. 2003, 15, 464-466.

12. Gao, P. X.; Song, J. H.; Liu, J.; Wang, Z. L. Nanowire Piezoelectric Nanogenerators on Plastic Substrates as Flexible Power Sources for Nanodevices. Adv. Mater. 2007, $19,67-72$.

13. Gao, X.; Li, X.; Yu, W. Flowerlike ZnO Nanostructures via Hexamethylenetetramine-Assisted Thermolysis of ZincEthylenediamine Complex. J. Phys. Chem. B 2005, 109, 1155-1161.

14. Wu, C. F. J.; Mao, S. S.; Ma, F. S. SEL: A Search Method Based on Orthogonal Arrays. In Statistical Design and Analysis of Industrial Experiments; Ghosh, S., Ed.;, Marcel Dekker: New York, 1990; pp 279-310.

15. Wu, C. F. J.; Hamada, M. Experiments: Planning, Analysis, and Parameter Design Optimization; Wiley: New York, 2000.

16. Xu, S.; Lao, C. S.; Weintraub, B.; Wang, Z. L. DensityControlled Growth of Aligned ZnO Nanowire Arrays by Seedless Chemical Approach on Smooth Surfaces. J. Mater. Res. 2008, 23, 2072-2077.
17. Fujibayashi, T.; Matsui, T.; Kondo, M. Improvement in Quantum Efficiency of Thin Film Si Solar Cells Due to the Suppression of Optical Reflectance at Transparent Conducting Oxide/Si Interface by $\mathrm{TiO}_{2} / \mathrm{ZnO}$ Antireflection Coating. Appl. Phys. Lett. 2006, 88, 183508.

18. Striemer, C. C.; Fauchet, P. M. Dynamic Etching of Silicon for Broadband Antireflection Applications. Appl. Phys. Lett. 2002, 81, 2980-2982.

19. Hu, L.; Chen, G. Analysis of Optical Absorption in Silicon Nanowire Arrays for Photovoltaic Applications. Nano Lett. 2007, 7, 3249-3252.

20. Lee, Y. J.; Ruby, D. S.; Peters, D. W.; McKenzie, B. B.; Hsu, J. W. P. ZnO Nanostructures as Efficient Antireflection Layers in Solar Cells. Nano Lett. 2008, 8, 1501-1505. 